\title{
The influence of creatine supplementation on the cognitive functioning of vegetarians and omnivores
}

\author{
David Benton $^{1 *}$ and Rachel Donohoe ${ }^{2}$ \\ ${ }^{1}$ Department of Psychology, University of Swansea, Swansea SA2 8PP, Wales, UK \\ ${ }^{2}$ Clinical Audit and Research Unit, London Ambulance Service NHS Trust, London, UK \\ (Received 3 February 2010 - Revised 12 October 2010 - Accepted 19 October 2010 - First published online 1 December 2010)
}

\begin{abstract}
Creatine when combined with P forms phosphocreatine that acts as a reserve of high-energy phosphate. Creatine is found mostly in meat, fish and other animal products, and the levels of muscle creatine are known to be lower in vegetarians. Creatine supplementation influences brain functioning as indicated by imaging studies and the measurement of oxygenated Hb. Given the key role played by creatine in the provision of energy, the influence of its supplementation on cognitive functioning was examined, contrasting the effect in omnivores and vegetarians. Young adult females ( $n$ 128) were separated into those who were and were not vegetarian. Randomly and under a double-blind procedure, subjects consumed either a placebo or $20 \mathrm{~g}$ of creatine supplement for $5 \mathrm{~d}$. Creatine supplementation did not influence measures of verbal fluency and vigilance. However, in vegetarians rather than in those who consume meat, creatine supplementation resulted in better memory. Irrespective of dietary style, the supplementation of creatine decreased the variability in the responses to a choice reaction-time task.
\end{abstract}

Key words: Attention: Creatine: Memory: Reaction times: Vegetarians

When creatine combines with $\mathrm{P}$ to form phosphocreatine, it acts as a reserve of high-energy phosphate that is used to rapidly convert ADP back to adenosine triphosphate ${ }^{(1)}$. In humans, although $95 \%$ of the total creatine pool is found in skeletal muscle, high levels are also found in the heart, brain and testes. In particular, it is surprising that the influence of brain creatine status has not attracted more interest as the brain is the most metabolically active organ in the body, accounting for $20 \%$ of BMR, although it represents only about $2 \%$ of body weight ${ }^{(2)}$. The present study has therefore examined the influence of creatine supplementation on human cognition, in particular comparing the response of vegetarians and omnivores as there are reports that a vegetarian diet is associated with poorer creatine status ${ }^{(3)}$.

A survey of the literature found only a few studies of the impact of creatine supplementation on cognitive functioning that have produced inconsistent findings. Rawson et $a l .{ }^{(4)}$ gave a creatine supplement to young adults for 6 weeks and found no influence on a battery of cognitive tests. However, McMorris et al. ${ }^{(5)}$ concluded that creatine supplementation aided cognition in the elderly when they found a significant effect of supplementation. Interestingly, Laakso et al. ${ }^{(6)}$ found that the level of creatine was significantly lower in the brain of those carrying apoE $\varepsilon 4$, a genetic risk factor for Alzheimer's disease, and that the level of brain creatine was related to memory problems. The authors speculated that creatine supplementation may be beneficial in those with the apoE $\varepsilon 4$ gene. Earlier McMorris et al. ${ }^{(7)}$ had reported in those who were sleep-deprived for $24 \mathrm{~h}$ that creatine supplementation had a positive effect on mood and cognition. A review ${ }^{(1)}$ concluded that in experimental paradigms of neurological diseases, supplementation with creatine can reduce neuronal loss. Adhihetty \& Beal ${ }^{(8)}$ similarly noted that a disruption of the provision of cellular energy is a characteristic of many neurodegenerative disorders, and that the use of creatine supplementation is beginning to be considered. They concluded that it is 'most efficacious as a treatment paradigm in Huntington's and Parkinson's disease'. Consistent with these findings, Rawson et al. ${ }^{(4)}$ suggested that creatine supplementation might only be beneficial in those with impaired cognitive processing.

As creatine can be synthesised by the body from the amino acids glycine, arginine and L-methionine, it is not an essential nutrient, and it has been suggested that most of the creatine in the brain is synthesised in situ ${ }^{(9)}$. There are, however, several reasons to suggest that neural activity may be susceptible to creatine supplementation. Using quantitative localised proton magnetic resonance spectroscopy, the 
consumption of $5 \mathrm{~g}$ of creatine monohydrate four times a day for a month resulted in an average increase of $8.7 \%$ in brain creatine levels ${ }^{(10)}$. Similarly the consumption of $8 \mathrm{~g}$ of creatine/d for $5 \mathrm{~d}$ resulted in decreased levels of oxygenated $\mathrm{Hb}$ in the brain: a response interpreted as evidence that increased oxygen utilisation had occurred while performing a demanding cognitive task for $15 \mathrm{~min}^{(11)}$. Over time, the decrease in the performance of the task was less in those who had consumed a creatine supplement.

A question that has not been adequately considered is the influence of a vegetarian diet, as creatine is found mostly in meat, fish and other animal products, with only a trace in some plants. The levels of muscle creatine are lower in vegetarians, leading to the suggestion that in terms of exercise they are likely to benefit to a greater extent from loading with creatine ${ }^{(12)}$. For example, Burke et $a l .{ }^{(3)}$ examined the levels of muscle creatine and exercise performance after supplementation. At baseline, muscle biopsy found lower levels of creatine in vegetarians. Following supplementation, the levels increased more in vegetarians than omnivores, a rise associated with an increased ability to exercise. Although there is an isolated suggestion that creatine improved the cognitive performance of vegetarians ${ }^{(13)}$, to date no study has systematically related the response to supplementation to the diet consumed. The present study therefore predicted a differential response to creatine supplementation in those who were and were not following a vegetarian diet.

\section{Methods}

\section{Subjects}

When they responded to a poster, potential subjects indicated whether they were vegan, vegetarian or if they ate meat. Female undergraduates ( $n$ 121), mean age $20 \cdot 3$ (SE $2 \cdot 1$ ) years, were recruited. None were consuming a creatine supplement, and all reported that they were in good health. Two groups were established: meat-eaters ( $n$ 51) and those who were vegan or vegetarian $(n 70)$. All subjects completed the trial. The study was conducted according to the guidelines laid down in the Declaration of Helsinki, and all procedures were approved by the Ethics Committee of Swansea University. Written informed consent was obtained from all subjects.

\section{Supplements}

Randomly and under a double-blind procedure, the subjects consumed either a placebo (sixty subjects) or a creatine (sixty-one subjects) supplement. Creatine monohydrate, $20 \mathrm{~g} / \mathrm{d}$, was consumed as $5 \mathrm{~g}$ tablets (Isostar Creatine; Wander Limited, King's Langley, Hertfordshire, UK). The subjects consumed the tablets at home and were asked to space their consumption throughout the day, in an empty stomach to facilitate absorption. The placebo was a glucose tablet of a similar size and appearance. Although there are suggestions that there may be a cognitive benefit from consuming glucose ${ }^{(14)}$, any response is short term and hence would not impact on the measures obtained under the present conditions. The tablets were consumed for a final time the evening before cognitive testing took place for a second occasion the following morning.

\section{Procedure}

A battery of cognitive tests was completed twice: before and after consuming the supplements. The supplements were consumed for $5 \mathrm{~d}$. The subjects were asked to return any tablets that had not been consumed which established that compliance in this relatively short-term study did not appear to be a problem. Although diet was not specifically monitored over the $5 \mathrm{~d}$ of the study, before testing, it was again established that those who had initially reported that they were vegetarian had avoided meat during the study.

\section{Cognitive tests}

Memory - word recall. Two lists of thirty nouns were created; all words had six letters and two syllables, chosen to be high in imagery, concreteness and frequency of use ${ }^{(15)}$. They were presented at the rate of one word per $2 \mathrm{~s}$ using a tape recorder. Immediately after presentation, the subjects wrote down as many words as they could recall in $2 \mathrm{~min}$. The number correctly recalled is reported.

\section{Reaction times}

The reaction-time procedure was based on that of Jensen ${ }^{(16)}$. On a panel, eight lamps were arranged in a semicircle $140 \mathrm{~mm}$. from a central button (the home key). The index finger was placed on the home key. There was a $0.5 \mathrm{~s}$ delay followed by a warning tone after which there was a further random delay between 1 and $4 \mathrm{~s}$, after which one of the eight lamps was illuminated. The subjects then, as quickly as possible, moved the hand from the home key to a button below the light, which when pressed extinguished it. The decision time is the delay between a light being illuminated and the hand being removed from the home key. Movement times were measured as the time taken for the hand to leave the home key and to press the button beneath the illuminated light.

Following a practice session of ten trials using all eight lamps, simple reactions were assessed when only one lamp could be illuminated. Choice reaction times were then recorded when two, four or eight lamps could be illuminated. In total, forty trials were run when each of one, two, four and eight lights were used. As recommended by Jensen $^{(16)}$, decision times were Winsorised to remove outliers; those scores outside the normal range of 170-990 ms and outside three standard deviations. 


\section{Vigilance - rapid information processing task}

A computer generated a series of digits, at the rate of 100 digits/min for $5 \mathrm{~min}$. The subjects pressed the space bar when they detected target sequences of three consecutive odd, or three consecutive even, digits. Eight of these sequences were presented every minute. Following the presentation of the third digit in a target sequence, $1500 \mathrm{~ms}$ was allowed for a correct response. A response under other circumstances was recorded as incorrect.

\section{Verbal fluency}

The Controlled Oral Word Association Test ${ }^{(17)}$ requires that subjects name as many words as possible, within $1 \mathrm{~min}$, beginning with a given letter of the alphabet, excluding proper nouns, numbers and the same word with a different suffix. Before consuming the supplements, the subjects were given the letters $\mathrm{C}, \mathrm{F}$ and $\mathrm{L}$ and after consuming the supplements, they were given the letters $\mathrm{P}, \mathrm{R}$ and $\mathrm{W}$. The score analysed was the sum of all acceptable words.

\section{Side effects}

At the end of the study, the subjects were asked if they had experienced any side effects and to guess whether they had consumed creatine or the placebo.

\section{Statistical analysis}

The data were analysed with appropriate ANOVA designs. The basic approach when only one outcome measure was examined was a three-way ANOVA: supplement (placebo/ creatine $) \times$ diet (vegetarian/meat eater) $\times$ time (before $/$ after supplement), with the last factor as a repeated measure. Additional factors, such as the number of lights to which responses were made in the reaction-time test, were added when necessary. Where a significant interaction resulted, it was examined by calculating simple and simple-simple main effects. Preliminary analysis found that those who were vegetarian as opposed to meat-eaters, and those who subsequently consumed the placebo as opposed to creatine supplement, did not differ on the baseline performance of any test.

\section{Results}

\section{Memory}

Fig. 1 illustrates that creatine influenced the recall of the word lists: the interaction supplement $\times$ diet $\times$ time reached significance $(F(1,117)=6.41 ; P<0.01)$. Before the supplement was consumed, the memory of vegetarians and meat-eaters was similar, irrespective of whether they were about to consume the placebo $(F(1,118)=0.93$; NS) or the creatine supplement $(F(1,118)=0.93$; NS $)$. After consuming the tablets for $4 \mathrm{~d}$, the memory of

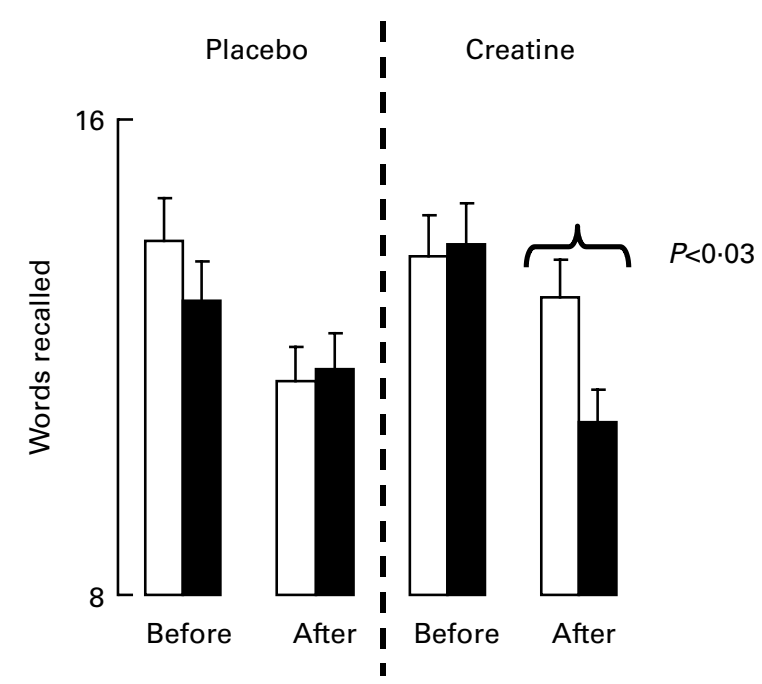

Fig. 1. The influence of creatine supplementation on memory. Values are the number of words recalled, before and after supplementation, as means with their standard errors represented by vertical bars. $\square$, Vegetarian; $\mathbf{\square}$, meat-eater.

vegetarians and meat-eaters was similar if they consumed the placebo $(F(1,118)=0 \cdot 01 ; \mathrm{NS})$. However, after $4 \mathrm{~d}$ of consuming the creatine supplement, memory was better in vegetarians rather than in those who consumed meat $(F(1,118)=5.06 ; P<0.03)$. However, in those who were meat-eaters, the consumption of the creatine supplement was associated with poorer memory compared with baseline values $(F(1,117)=17 \cdot 84 ; P<0 \cdot 001)$.

\section{Reaction times}

Neither decision times (tablet $\times$ before/after, $F(1,117)=$ $0 \cdot 11$; NS) nor movement times $(F(1,117)=0 \cdot 16$; NS $)$ were influenced by the supplementation of creatine or by the dietary style (Table 1 ). However, variability in the speed of response is known to be a more sensitive measure, and therefore the standard deviations of the decision times were analysed: the interaction supplement $\times$ number of lamps monitored $\times$ time reached statistical significance $(F(3,351)=2.59 ; P<0.05)$. Fig. 2 illustrates the effect that reflected differences when eight but not fewer lamps were monitored. In those who were consuming the placebo, performance was more variable when tested a second time $(F(1,119)=6.58 ; P<0.01)$, although after consuming the creatine supplement it was associated with a similar performance on both occasions $(F(1,19)=$ 0.03 ; NS). This effect occurred irrespective of whether the subject was a vegetarian.

\section{Vigilance}

Supplementation did not influence the ability to sustain attention. Neither the number of correct (supplement $\times$ diet $\times$ time, $F(1,117)=3 \cdot 56$; NS) nor the number of incorrect responses (supplement $\times$ diet $\times$ time, $F(1,117)=0.60$; NS) reached statistical significance (Table 1 ). 
Table 1. Influence of creatine supplementation on measures of cognition (Mean values with their standard errors)

\begin{tabular}{|c|c|c|c|c|c|c|c|c|}
\hline & \multicolumn{2}{|c|}{ Before tablets } & \multicolumn{2}{|c|}{ After tablets } & \multicolumn{2}{|c|}{ Before tablets } & \multicolumn{2}{|c|}{ After tablets } \\
\hline & Mean & SE & Mean & SE & Mean & SE & Mean & SE \\
\hline \multicolumn{9}{|c|}{ Decision times* } \\
\hline \multicolumn{9}{|c|}{1 Lamp } \\
\hline Placebo & 293 & $9 \cdot 2$ & 299 & $8 \cdot 2$ & 296 & $7 \cdot 0$ & 298 & $6 \cdot 2$ \\
\hline Creatine & 305 & $8 \cdot 1$ & 302 & $7 \cdot 1$ & 303 & $7 \cdot 7$ & 300 & $6 \cdot 8$ \\
\hline \multicolumn{9}{|l|}{2 Lamps } \\
\hline Placebo & 324 & $7 \cdot 7$ & 324 & $9 \cdot 8$ & 317 & $5 \cdot 8$ & 315 & $7 \cdot 5$ \\
\hline Creatine & 324 & $6 \cdot 7$ & 326 & $8 \cdot 6$ & 324 & $6 \cdot 4$ & 328 & $8 \cdot 1$ \\
\hline \multicolumn{9}{|l|}{4 Lamps } \\
\hline Placebo & 352 & $9 \cdot 0$ & 359 & $8 \cdot 7$ & 345 & $6 \cdot 8$ & 350 & $6 \cdot 6$ \\
\hline Creatine & 355 & $7 \cdot 8$ & 356 & $7 \cdot 6$ & 350 & $7 \cdot 4$ & 353 & $7 \cdot 2$ \\
\hline \multicolumn{9}{|l|}{8 Lamps } \\
\hline Placebo & 402 & $15 \cdot 8$ & 399 & $13 \cdot 7$ & 407 & $12 \cdot 0$ & 409 & $10 \cdot 4$ \\
\hline Creatine & 414 & $13 \cdot 8$ & 419 & $12 \cdot 0$ & 409 & $13 \cdot 1$ & 427 & 11.4 \\
\hline \multicolumn{9}{|c|}{ Movement times* } \\
\hline \multicolumn{9}{|c|}{1 Lamp } \\
\hline Placebo & 228 & $10 \cdot 0$ & 255 & $11 \cdot 6$ & 223 & $7 \cdot 6$ & 230 & 8.9 \\
\hline Creatine & 237 & 8.7 & 240 & $10 \cdot 2$ & 224 & $8 \cdot 3$ & 225 & $9 \cdot 7$ \\
\hline \multicolumn{9}{|l|}{2 Lamps } \\
\hline Placebo & 227 & 8.5 & 241 & $11 \cdot 3$ & 214 & $6 \cdot 5$ & 230 & $8 \cdot 6$ \\
\hline Creatine & 233 & $7 \cdot 4$ & 240 & $9 \cdot 8$ & 224 & $7 \cdot 1$ & 231 & $9 \cdot 3$ \\
\hline \multicolumn{9}{|l|}{4 Lamps } \\
\hline Placebo & 229 & $8 \cdot 9$ & 243 & $12 \cdot 3$ & 220 & $6 \cdot 8$ & 229 & $9 \cdot 4$ \\
\hline Creatine & 296 & $7 \cdot 7$ & 245 & $10 \cdot 8$ & 231 & $7 \cdot 4$ & 248 & $10 \cdot 3$ \\
\hline \multicolumn{9}{|l|}{8 Lamps } \\
\hline Placebo & 235 & $9 \cdot 6$ & 252 & $12 \cdot 9$ & 226 & $7 \cdot 3$ & 237 & $9 \cdot 8$ \\
\hline Creatine & 245 & $8 \cdot 4$ & 253 & $11 \cdot 3$ & 237 & $8 \cdot 0$ & 246 & $10 \cdot 7$ \\
\hline \multicolumn{9}{|l|}{ Vigilance† } \\
\hline \multicolumn{9}{|l|}{ Correct } \\
\hline Placebo & $19 \cdot 2$ & $1 \cdot 3$ & $22 \cdot 1$ & 1.4 & $20 \cdot 2$ & $1 \cdot 0$ & $22 \cdot 5$ & $1 \cdot 1$ \\
\hline Creatine & $18 \cdot 7$ & $1 \cdot 1$ & $22 \cdot 1$ & $1 \cdot 3$ & $19 \cdot 7$ & $1 \cdot 1$ & $21 \cdot 5$ & $1 \cdot 2$ \\
\hline \multicolumn{9}{|l|}{ Incorrect } \\
\hline Placebo & $4 \cdot 0$ & 0.6 & 4.5 & $1 \cdot 1$ & 2.9 & 0.5 & 1.9 & 0.8 \\
\hline Creatine & 2.9 & 0.6 & 3.6 & $1 \cdot 0$ & $3 \cdot 3$ & 0.6 & 3.4 & 0.9 \\
\hline \multicolumn{9}{|c|}{ Verbal fluency $\ddagger$} \\
\hline Placebo & $36 \cdot 6$ & $2 \cdot 1$ & $40 \cdot 2$ & $2 \cdot 0$ & $37 \cdot 2$ & $1 \cdot 6$ & $39 \cdot 0$ & 1.6 \\
\hline Creatine & $36 \cdot 0$ & $1 \cdot 8$ & $40 \cdot 9$ & $1 \cdot 8$ & $37 \cdot 2$ & $1 \cdot 7$ & $40 \cdot 5$ & $1 \cdot 7$ \\
\hline
\end{tabular}

* With decision and movement times, the values are in $\mathrm{ms}$.

t With vigilance, the number of correct sequences observed and inappropriate responses are reported.

$\ddagger$ The total number of words reported is the measure of verbal fluency.

\section{Word fluency}

Similarly, supplementation did not influence word fluency, with the interaction supplement $\times$ diet $\times$ time being non-significant $(F(1,117)=0 \cdot 21$; NS; Table 1$)$.

\section{Side effects}

When asked to guess which supplement they had consumed, of those consuming the placebo, $18.3 \%$ said creatine, $31.6 \%$ placebo and $50 \%$ did not know. These data were compared with those who received the active supplement of whom $14.8 \%$ said creatine, $24.6 \%$ placebo and $60 \%$ did not know. The use of $\chi^{2}$ demonstrated that these distributions were not significantly different. When asked whether they experienced side effects, $85 \%$ of those consuming the placebo and $73.8 \%$ of those after taking creatine said that they had experienced none. The distributions obtained, depending on the supplement consumed, did not differ significantly. The side effects were in all cases minor, for example reporting feeling bloated or having a headache. These data suggested that the blind was maintained throughout the study.

\section{Discussion}

The present findings supported the view that brain creatine status can influence psychological functioning, as supplementation influenced memory (Fig. 1) and decision times (Fig. 2) in at least some of the sample. The finding that the supplementation of creatine was associated with better memory (Fig. 1) is consistent with Rae et al. ${ }^{(13)}$ who when considering only vegetarians found that supplementation improved memory. The present findings raise various questions concerning the effect on memory. Overall, memory was poorer when assessed on the second occasion irrespective of whether the placebo or creatine supplement had been consumed, raising the 

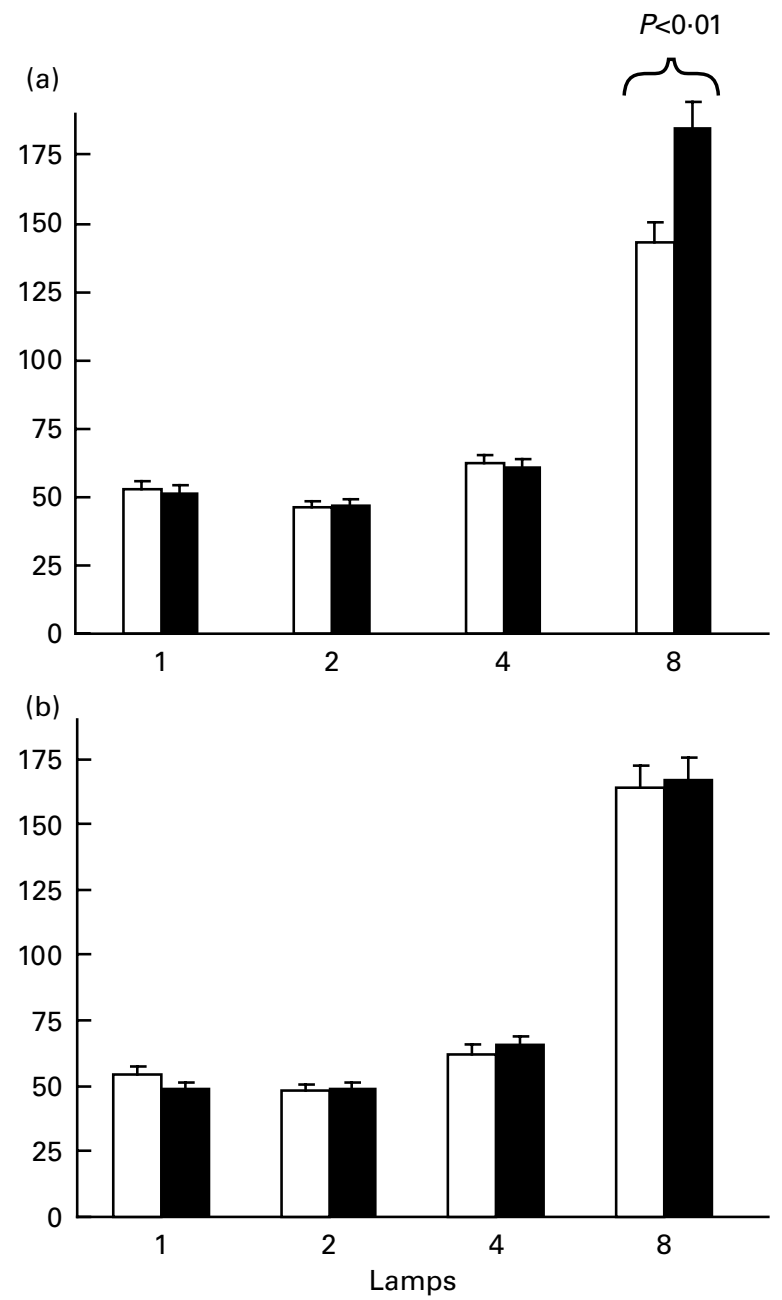

Fig. 2. The influence of creatine supplementation on the standard deviation of the decision times. Values are means of the standard deviations, with standard errors represented by vertical bars when various numbers of lamps were monitored. (a) Placebo ( $\square$, before tablets; $\mathbf{\square}$, after tablets). (b) Creatine.

possibility that unexpectedly the second word list was more difficult than the first, although the way in which the two lists were matched argues against this as an explanation. Alternatively, there may have been a lack of motivation towards the end of the study, although the findings with the other cognitive measures do not support a generally more negative attitude. The major finding was that after supplementation, the memory of vegetarians was better than that of meat-eaters (Fig. 1). However, at baseline, memory did not differ depending on dietary style, so any hypothesised creatine deficiency in vegetarians did not influence memory, rather it was found that vegetarians were more sensitive to supplementation with creatine.

The finding that decision times were less variable after consuming the creatine supplement (Fig. 2) is consistent with the report ${ }^{(11)}$ that it reduced the rate at which a decline in the performance of a demanding task occurred. Although mean reaction times are often reported, there is a long tradition of considering intra-individual variability; in fact, Jensen ${ }^{(16)}$ considered this the most sensitive measure that can be derived from reaction times. Increased intra-individual variability is observed during ageing as well as traumatic brain injury, schizophrenia, attentiondeficit hyperactivity disorder and dementia ${ }^{(17)}$. Thus, intra-individual variability can be viewed as a behavioural marker of compromised neural functioning. MacDonald et $a l .{ }^{(18)}$ concluded that intra-individual variability reflects multiple neural determinants including the functioning of the frontal lobes. They suggested that various neurotransmitters, in particular catecholamine and acetylcholine, contribute to greater intra-individual variability in cognitive performance $^{(19)}$.

The results can be viewed as creatine supplementation selectively influencing a demanding task that placed greater physiological demands on the brain, as it was only the monitoring of eight lamps that benefited from creatine (Fig. 2). The consumption of the placebo was associated with a poorer performance when tested for a second time, a decline prevented by consuming the creatine supplement. That the nature of the pre-existing diet did not influence the response to supplementation with the reaction time task suggested a more general effect.

It is possible that the dose used influences the response of the brain. Athletes wishing to benefit from creatine supplementation are recommended to consume $5 \mathrm{~g}$ doses, four times a day, for 5-6d, after which a maintenance dose of $2-5 \mathrm{~g}$ a day is administered. Casey \& Greenhaff ${ }^{(20)}$ found no evidence that increasing the dose beyond $20 \mathrm{~g}$ a day, during this initial period, further increased creatine uptake or athletic performance. The dose used in the present study reflected that used in the previous study that has considered increases in the creatine content of muscle. It is possible that the rate at which levels increase in the brain differ from muscle, but McMorris et al. ${ }^{(7)}$ reported that following $24 \mathrm{~h}$ of sleep deprivation, a dose similar to that used in the present study improved mood and the performance of tasks that placed a heavy demand on the prefrontal cortex.

One can speculate about the underlying mechanism. Phosphocreatine can be expected to play a role in supplying energy to metabolically active areas of the brain. There are several isoforms of creatine phosphokinase, the enzyme that converts creatine to phosphocreatine. The most common variety is found on the outer surface of the inner mitochondrial membrane and is coupled to the adenine nucleotide translocator. In the brain, this mitochondrial type is found, but there is also a brain-type creatine phosphokinase. Visualisation of the brain-type creatine phosphokinase shows that it is found in the 'somata of Golgi type 1 neurons in the cerebellum, red nucleus and pons and is distributed throughout the cell body and within nuclei. Brainphosphokinase immunostaining also appears in neuronal processes and is concentrated in the molecular layers of the cerebellum and the hippocampus and in cortical pyramidal cell dendrites'; the mitochondrial type is found 'in the somata of all Golgi type 1 neurons in the cerebellum, 
pontine reticular formation, red nucleus, hippocampus and cerebral cortex... and appears throughout the cell body but not in nuclei ${ }^{\text {(21) }}$. As most of the brain-type creatine phosphokinase is found in neuronal processes, particularly in synaptic regions, a role in neurotransmission is suggested. The uneven distribution of creatine phosphokinase indicates that rather than having a general function, it is important in some aspects of functioning rather than others. The high concentration in the hippocampus is of particular interest, given the known importance of this area of the brain in memory. The previous report that creatine supplementation improves memory ${ }^{(4)}$ and the present finding that memory benefited from supplementation (Fig. 1) are consistent with these physiological data.

It is, however, possible that the effect of supplementation may be indirect. For example, in vegetarians supplemented with creatine, Rooney et al. ${ }^{(22)}$ found that glucose homeostasis was affected. In an oral glucose tolerance test, there was a greater glucose response, although it did not affect insulin levels. Given the repeated suggestions that a glucose drink and individual differences in glucose tolerance influence memory ${ }^{(23,24)}$, this is a mechanism that should be examined.

To date, the findings are too preliminary to allow the role of creatine in the brain and the impact of its supplementation on cognition to be established. However, the present and previous reports ${ }^{(11,13)}$ that supplementation influences cognitive functioning, and the evidence that creatine supplementation influences basic brain physiology ${ }^{(10,11)}$, suggest that the topic will repay further examination.

\section{Acknowledgements}

There are no conflicts of interests, and neither of the authors has any financial interest in the sale of creatine or creatine-containing products. The supplements were a gift from Wander Limited, King's Langley, Hertfordshire, UK, otherwise the study was not externally funded. The data were collected by R. D. who was responsible for the statistical analysis. D. B. designed the study and reported the findings.

\section{References}

1. Andres RH, Ducray AD, Schlattner U, et al. (2008) Functions and effects of creatine in the central nervous system. Brain Res Bull 76, 329-343.

2. Benton D (2005) Diet, cerebral energy metabolism and psychological functioning. In Nutrition, Brain and Behavior, vol. 3, Nutritional Neuroscience: Overview of an Emerging Field, pp. 57-71 [C Prasad, H Lieberman and R Kanarek, editors]. Boca Raton, FL: Taylor and Francis.

3. Burke DG, Chilibeck PD, Parise G, et al. (2003) Effect of creatine and weight training on muscle creatine and performance in vegetarians. Med Sci Sports Exerc 35, 1946-1955.
4. Rawson ES, Lieberman HR, Walsh TM, et al. (2008) Creatine supplementation does not improve cognitive function in young adults. Physiol Behav 95, 130-134.

5. McMorris T, Mielcarz G, Harris RC, et al. (2007) Creatine supplementation and cognitive performance in elderly individuals. Aging Neuropsychol Cogn 14, 517-528.

6. Laakso MP, Hiltunen Y, Kononen M, et al. (2003) Decreased brain creatine levels in elderly apolipoprotein E $\varepsilon 4$ carriers. J Neural Transm 110, 267-275.

7. McMorris T, Harris RC, Swain J, et al. (2006) Effect of creatine supplementation and sleep deprivation, with mild exercise, on cognitive and psychomotor performance, mood state, and plasma concentrations of catecholamines and cortisol. Psychopharmacology 185, 93-103.

8. Adhihetty PJ \& Beal MF (2008) Creatine and its potential therapeutic value for targeting cellular energy impairment in neurodegenerative diseases. Neuromolecular Med 10, $275-290$.

9. Wyss M \& Schulze A (2002) Health implication of creatine: can oral creatine supplementation protect against neurological and atherosclerotic disease? Neuroscience 112, 243-260.

10. Dechent P, Pouwels PJ, Wilken B, et al. (1999) Increase of total creatine in human brain after oral supplementation of creatine-monohydrate. Am J Physiol 277, R698-R704.

11. Watanabe A, Kato N \& Kato T (2002) Effects of creatine on mental fatigue and cerebral hemoglobin oxygenation. Neurosci Res 42, 279-285.

12. Barr SI \& Rideout CA (2004) Nutritional considerations for vegetarian athletes. Nutrition 20, 696-703.

13. Rae C, Digney AL, McEwan SR, et al. (2003) Oral creatine monohydrate supplementation improves brain performance: a double-blind, placebo-controlled, cross-over trial. Proc Biol Sci 270, 2147-2150.

14. Benton D \& Parker PY (1998) Breakfast, blood glucose and cognition. Am J Clin Nutr 67, 772S-778S.

15. Quinlan PT (1992) The Oxford Psycholinguistic Database. Oxford: Oxford University Press.

16. Jensen AR (1987) Individual differences in the Hick paradigm. In Speed of Information-processing and Intelligence, pp. 101-175 [PA Vernon, editor]. Norwood, NJ: Ablex.

17. Benton A \& Hamsher K (1976) Multilingual Aphasic Examination. Iowa City, IA: University of Iowa.

18. MacDonald SW, Li SC \& Bäckman L (2009) Neural underpinnings of within-person variability in cognitive functioning. Psychol Aging 24, 792-808.

19. MacDonald SW, Nyberg L \& Bäckman L (2006) Intraindividual variability in behavior: links to brain structure, neurotransmission and neuronal activity. Trends Neurosci 29, 474-480.

20. Casey A \& Greenhaff PL (2000) Does dietary creatine supplementation play a role in skeletal muscle metabolism and performance? Am J Clin Nutr 72, 607S-617S.

21. Friedman DL \& Roberts R (1994) Compartmentation of braintype creatine kinase and ubiquitous mitochondiral creatine kinase in neurons: evidence for a creatine phosphate energy shuttle in adult rat brain. J Comp Neurol 343, 500-511.

22. Rooney KB, Bryson JM, Digney AL, et al. (2003) Creatine supplementation affects glucose homeostasis but not insulin secretion in humans. Ann Nutr Metab 47, 11-15.

23. Benton D \& Owens D (1993) Blood glucose and human memory. Psychopharmacology 113, 83-88.

24. Donohoe RT \& Benton D (2000) Glucose tolerance predicts performance on tests of memory and cognition. Physiol Behav 71, 395-401. 